\title{
Adaptive robotic end-effector with embedded 3D-printed sensing circuits
}

\author{
Aurelian Zapciu ${ }^{1, *}$, George Constantin ${ }^{1}$, and Diana Popescu ${ }^{1}$ \\ ${ }^{1}$ University POLITEHNICA of Bucharest, Department of Machine and Production Systems, 313 \\ Splaiul Independentei, sector 6, Bucharest, Romania
}

\begin{abstract}
The paper presents the development and testing of an adaptive robotic end-effector used for manipulation of sensitive objects such as fruits and vegetables. The end-effector uses Fin-Ray-structured 3D-printed fingers with embedded conductive 3D-printed sensing circuits, which give the end-effector capacitive touch sensing and bend sensing capabilities. The conductive 3D-printed circuit is connected to a control circuit consisting of a low-current DC power source and a microcontroller. As the end-effector finger is subjected to various forces and other external stimuli, changes in the electric signals that run through the conductive circuit of the end-effector finger are detected by the microcontroller. The electric signal is processed in order to provide real-time information about contact detection, finger position or gripping force. This information was used for process monitoring purposes and as feedback for the end-effector actuator.
\end{abstract}

\section{Introduction}

Research in the field of additive manufacturing (AM) focused recently on improving materials and processes. Thus, processes, such as PolyJet, allowing the build of realistic multi-color, multi-materials parts [1] were developed for use in many fields, especially for visualization, marketing or training purposes, but improvements were as well brought to the existing AM processes in terms of accuracy, resolution, reproducibility, adaptability to new materials, printed part quality and mechanical properties [2]. Currently 3D-printing can produce flexible metamaterial parts [3], parts with controlled microstructures and properties [4] for smart materials applications, as well as functional parts and assemblies from materials with electric current conductivity that can be used in a large range of applications and industries [5].

Robotics is one field which benefits from these advances. The possibility to 3D print intelligent end-effectors that can adapt to objects' shapes and dimensions, similar to a human hand, opens new perspectives in manipulating, for instance, sensitive or irregular objects like fruits or vegetables, as the universal gripper based on jamming granular material [6] does. In this context, this study is focused on developing and testing an endeffector with three 3D-printed fingers based on Fin-Ray structures [7-8]. It embeds 3Dprinted conductive sensing circuits, which provide the end-effector capacitive touch sensing and bend sensing capabilities.

\footnotetext{
*Corresponding author: aurelianzapciu@yahoo.com
} 
Adaptive or soft grippers are designed and optimized for different tasks as presented in [9], their ability to adapt to vary surfaces and sizes offering the versatility required in diverse applications ranging from grabbing biological samples [10] to thumb rehabilitation [11]. Although all based on Fin-Ray effect, the differences in design (dimensions, number of fingers, number and type of joints, etc.) determine different grip strength which is an important factor to consider as it is highly related to the end-effector functionality and application constraints. Moreover, there are different actuation solutions and modalities to monitor the fingers position and the contact between object and gripper, our solution being based on establishing a control circuit between the conductive 3D-printed circuit in each finger, the power source and a microcontroller. The changes in electric signals when the finger is subjected to a load are detected in real time by the microcontroller and used as feedback for the actuator.

The use of Fused Deposition Modeling (FDM) process for manufacturing end-effector fingers with embedded conductive sensing Circuits already proved feasibility [12], this research focusing on developing and testing a whole gripper.

\section{Method and materials}

The multiple approaches to gripper adaptability presented previously, while functionally sound, experience difficulties when it comes to accessing data from the environment and providing feedback. To address this, third-party vision or sensor systems are used driving up system complexity and costs.

The approach taken by the authors uses novel conductive polymers to create electrically conductive circuits which are integrated in end-effector parts using multi-material 3D printing. Previous work [12] focused on identifying methods and physics which could be applied in the development of such an end-effector, while the current paper considers integrating multiple of these principles in a single prototype.

First, the experimental adaptive end-effector (fig. 1a) under development integrates capacitive touch sensors, which are used to sense the contact between the fingers and the target object. This effect is achieved using a plate made out of conductive polymer, which forms the electrode of a capacitor, fabricated on top of an insulating plate. When a target object enters this electrode's electrostatic field, it changes its capacitance. The second capability is sensing the bending of the end-effector finger under load. As the material bends, the outer radius of a conductive plate becomes longer or shorter than its nominal length under no load. The conductive polymer is subjected to elongation or compression

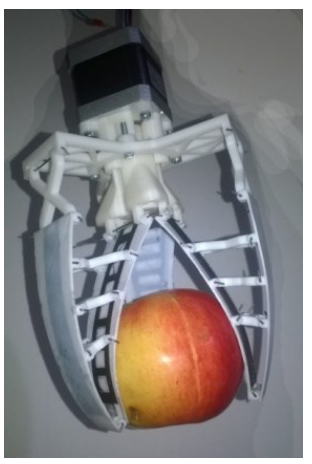

a

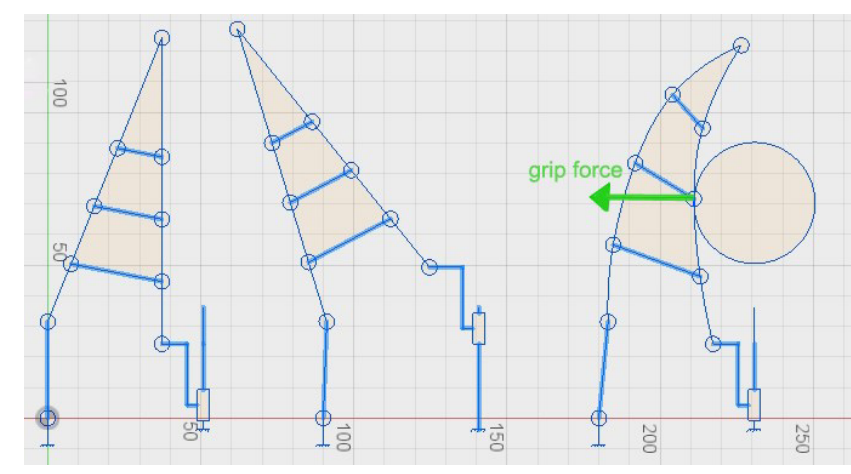

$\mathrm{b}$

Fig. 1. Developed adaptive end-effector: $a$-end-effector during testing; $b$ - end-effector geometry and forces acting on a finger. 


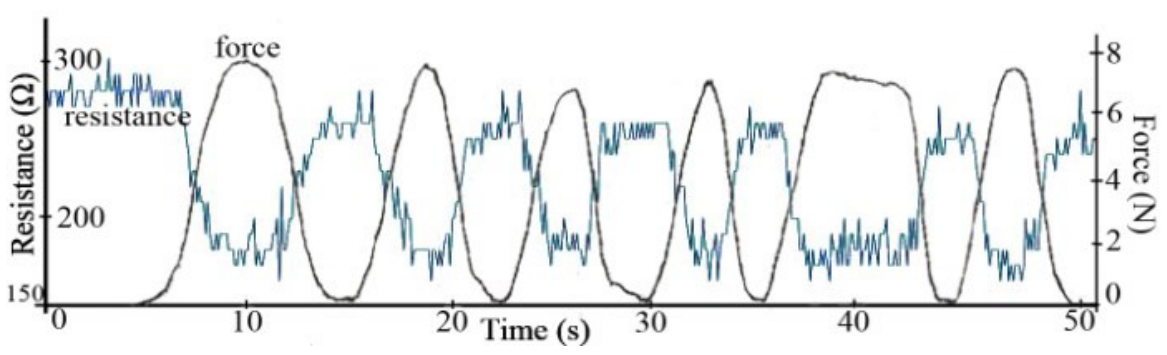

Fig. 2. Resistance of a 3D printed and applied force depending on time.

strain and microscopic cracks form in the structure, lowering or raising the resistivity of the conductive circuit. This effect is visible in fig. 2 showing resistance, which decreases when the gripping force of an end-effector finger increases.

\subsection{Materials}

Throughout the experiments, four different materials were used for manufacturing the gripper parts and the parts for the experimental stand. Unfilled ABS polymer filament was used to create the structure of the end-effector fingers and form the insulating plates of the integrated capacitive and bending sensors. For the conductive parts of the end-effector fingers, using an ABS-based material allows for a strong bond between part layers and prevents delamination during bending. Another important aspect of multi-material 3D printing is thermal expansion coefficient compatibility, as too big of a difference between the thermal expansion coefficients of different materials can create internal mechanical strain which can destroy the part during the cooling process. The chosen material was 3D Prima's Conductive ABS. The dual-material parts were fabricated using a QidiTech Avatar IV 3D printer. For the other components of the testing device, which have higher geometrical complexity and require higher dimensional accuracy, ABS P400 and ABS P400 Support were used. These components were fabricated using a Mojo 3D printer (Stratasys).

\subsection{Determining a suitable build orientation}

Considering how loads are applied in gripping applications and the geometry of the endeffector finger (fig. 1b) it was concluded that the ideal part orientation during fabrication is horizontal. However, given that electrical properties also vary with layer orientation, and for this application the sensing capabilities of the final product are just as important as mechanical strength, it was determined that it was necessary to test the second best part orientation as well, which is in the vertical plane, with horizontal layers covering the length of the part.

In order to determine which part orientation allows for the optimal electrical properties of this application, a series of four $80 \times 15 \times 4 \mathrm{~mm}$ test parts (fig. $3 \mathrm{a}$ ) were fabricated and then tested. The test parts were orientated so they can be fabricated without the use of support material, with $0.8 \mathrm{~mm}$ thick conductive material plates orientated in the horizontal or in vertical plane. The conductive plates are insulated using a $0.4 \mathrm{~mm}$ thick unfilled ABS layer. For the parts fabricated in the vertical plane, the conductive layers were printed using parallel filament strands along the length of the part (fig. 3b) or in a zigzag pattern (fig. 3c). For the parts fabricated in the horizontal plane, the conductive layers were printed using an orthogonal crosshatch pattern with parallel (fig. $3 \mathrm{~d}$ ) and $45^{\circ}$ (fig. $3 \mathrm{e}$ ) road orientations. 


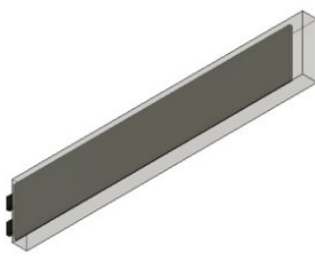

a

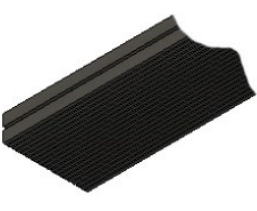

b

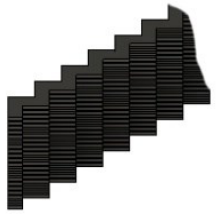

C

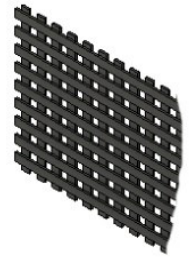

d

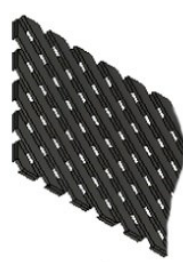

e

Fig. 3. Patterns used to test build orientation: $a$ - test parts; $b$-parallel filament; $c$-zigzag pattern; $d$ - crosshatch parallel; $e$ - crosshatch $45^{\circ}$ orientation.

Test parts were connected to an Arduino UNO microcontroller. The resistance of the conductive circuit is determined using the controller's integrated voltmeter and a simple voltage divider circuit where the measured resistance is wired in series with a known-value resistor. The known resistor is sized to match the resistance of the conductive circuit under no load, which was measured manually. The capacitive sensing capabilities are tested using a timing circuit that uses the controller's integrated timer and a $5 \mathrm{M} \Omega$ resistance. Capacitance variance is tracked using arbitrary units, as determining the absolute value of the capacitance is not needed in this touch-sensitive application.

In order to test the integrated bending sensor, the parts were bent on a testing rig (fig. 4). Resistance was measured for each $5 \mathrm{~mm}$ increments in displacement in the median plane between 0 and $20 \mathrm{~mm}$. 5 sets of measurements were taken and the values were averaged.

The results of this preliminary test are shown in fig. 5. The test samples that were fabricated with the conductive layers in the vertical plane (square and star markers) performed poorly. The resistance of the conductive circuit does not vary sufficiently to provide a reliable determination of finger bending. The test samples that were fabricated in the horizontal plane both provided satisfactory results. The conductive circuit printed with an angular orthogonal pattern (up-pointing triangle) is preffered over the one that is parallel with the length of the part (down-pointing triangle) as the experiment show a bigger drop in resistance.

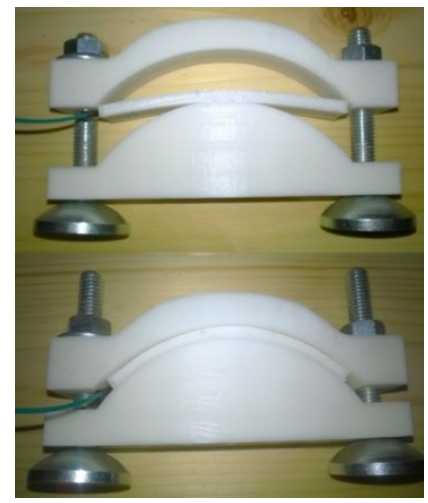

Fig. 4. Experimental stand for bending tests of finger plates.

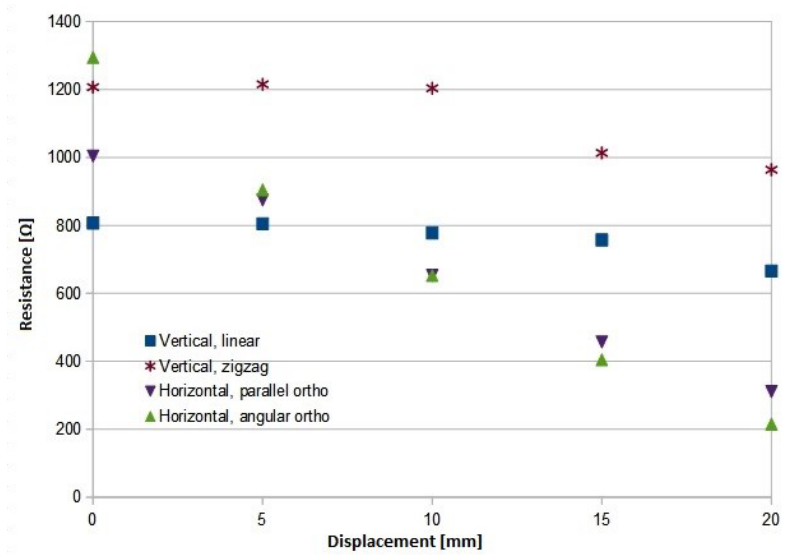

Fig. 5. Results of the bending test (vertical linear, fig.3b; vertical zigzag, fig. $3 \mathrm{c}$; horizontal parallel orthogonal; fig. $3 \mathrm{~d}$, horizontal angular orthogonal, fig.3e). 


\subsection{Adaptive gripper prototype design}

A prototype for an end-effector was designed using Autodesk 360 Fusion (fig. 6). The prototype uses three Fin Ray-type fingers, each composed of two sides with integrated conductive circuits. In total, six different conductive circuit patterns were designed and 3D printed. As determined from previous testing, the end-effector finger components were printed in a horizontal orientation, using orthogonal cross-hatch at a $45^{\circ}$ angle. Each configuration was printed in a single process, using a dual-extruder QidiTech Avatar IV 3D printer.

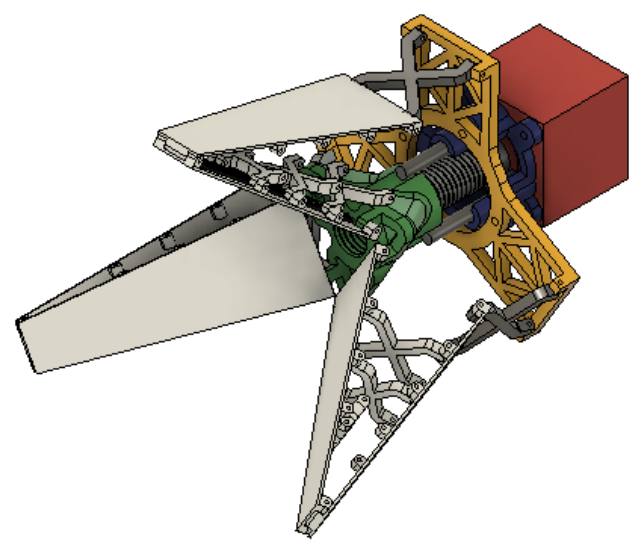

Fig. 6. Virtual prototype of the adaptive gripper.

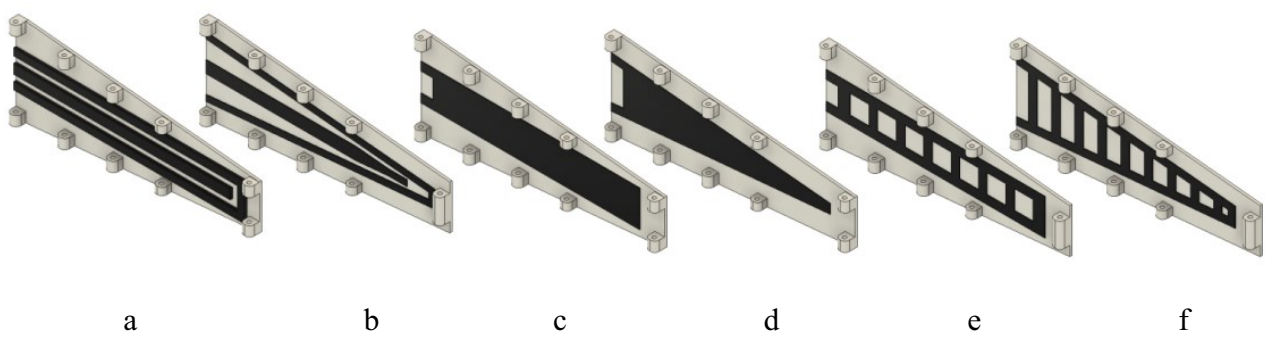

Fig. 7. Various conductive plate designs 3D printed: $a-\mathrm{v} 1$, parallel stripes; $b-\mathrm{v} 2$, inclined stripes; $c-\mathrm{v} 3$, rectangular area; $d-\mathrm{v} 4$, triangular area; $e-\mathrm{v} 5$, rectangular ladder; $f-\mathrm{v} 6$, trapezoidal ladder.

\subsection{Experimental setup}

The testing setup is shown in fig. 8. The three Fin-Ray end-effector fingers were connected using steel wire to the main body which has an internal M16 thread. A 3D printed screw fixed to the shaft of the stepper motor drives the main body. The main body slides along a system of three steel round guides.

The end-effector prototype has a maximum opening of $110 \mathrm{~mm}$ at the tip of the fingers and $30 \mathrm{~mm}$ at the base. It can grasp firm round objects up to $80 \mathrm{~mm}$ in diameter and soft objects up to $100 \mathrm{~mm}$ in diameter. A stepper motor is connected to an Allegro A4988 stepper motor driver and powered by a $12 \mathrm{~V}$ DC power supply. The entire system is controlled by an Arduino UNO controller connected to a laptop computer. The controller is powered through the USB port of the laptop computer. Data retrieved by the controller is sent via serial protocol to the laptop computer, plotted in real-time on the screen and saved in CSV format for further analysis. For this particular prototype, a stepper motor drive was preferred due to the simplicity of controlling it using the Arduino UNO controller, but all 
the results presented in this paper can be replicated with pneumatic or other types of actuators as well, as the sensing system does not depend on the driving method.

In order to determine which conductive circuit design performs better and to verify the feasibility of applying this concept in a real-world setting, a 12-hour endurance test was carried. The gripper is closed to grasp a tennis ball. The grasp is held for 2 seconds, after which the gripper is opened and the tennis ball is released. After 1 second, the test cycle is repeated. In total, one cycle lasts 8 seconds. After 12 hours and more than 5,000 test cycles, the orientations of the three end-effector fingers are reversed and another 12-hour test is carried. In total, each conductive circuit design was tested for 5,000 cycles while positioned on the inside part of the gripper finger (on the face that comes in contact with the target object) and 5,000 cycles while positioned on the outside part.

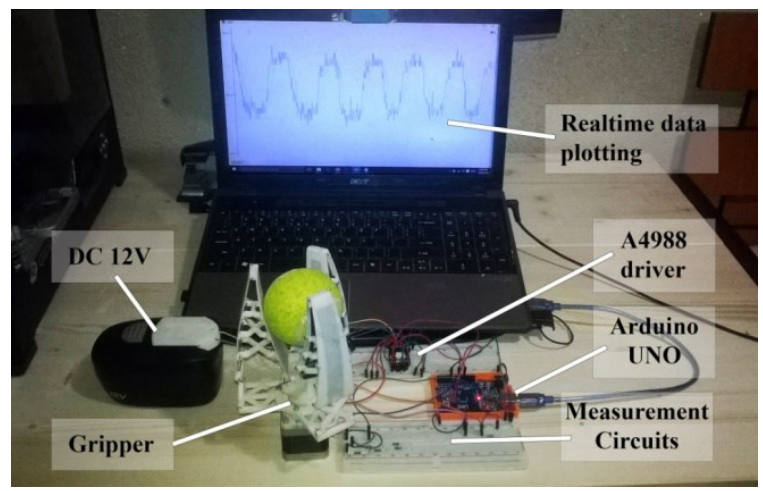

Fig. 8. Experimental setup for testing the adaptive end-effector prototype.

\section{Results}

Four out of the 6 conductive circuit designs - v1, v2, v5, v6 -showed good repeatability during the 24-hour continuous testing (more than 10,000 work cycles). Figure 9 shows the changes in the resistance of these 4 bend sensor designs during a work cycle. For better visualization of the performance, the graphs show the measured resistance increase or decrease in relative percentages, $0 \%$ being the resistance of the unloaded conductive circuit.

Results show that placing the finger face which contains the embedded bend sensor towards the target object produces an increase of resistance of up to $100 \%$ the initial value, allowing for a proportional determination of the amount the finger bends. When the bend sensor is positioned on the face that is away from the target object, there is a drop in resistance which is nominally less significant and the results are harder to use.

Following the endurance tests, an automated grasping procedure was setup using a feedback loop from the two embedded sensing circuits. Signal input from the capacitive touch sensor is used as a trigger to start closing the gripper and signal input from the bend sensor is used to stop the closing process when a threshold is met. The design variant chosen for this test was $\mathrm{v} 1$, which has shown the most reliable performance during the endurance test. The finger is mounted with the bend sensor on the inner face, as this positioning showed the biggest resistance difference. To further increase the reliability of the bend sensor readings, a signal smoothing algorithm was used (moving average). For this test, an apple attached to a string was used. The apple is lowered towards the opened gripper. When the apple touches the surface of the fingers, the change in capacitance is sensed and the microcontroller commands the stepper motor to close the gripper. As the gripper closes, the fingers start bending around the apple. When signal from the bend sensor reaches the preset threshold, the stepper is stopped and the grasp is held. 

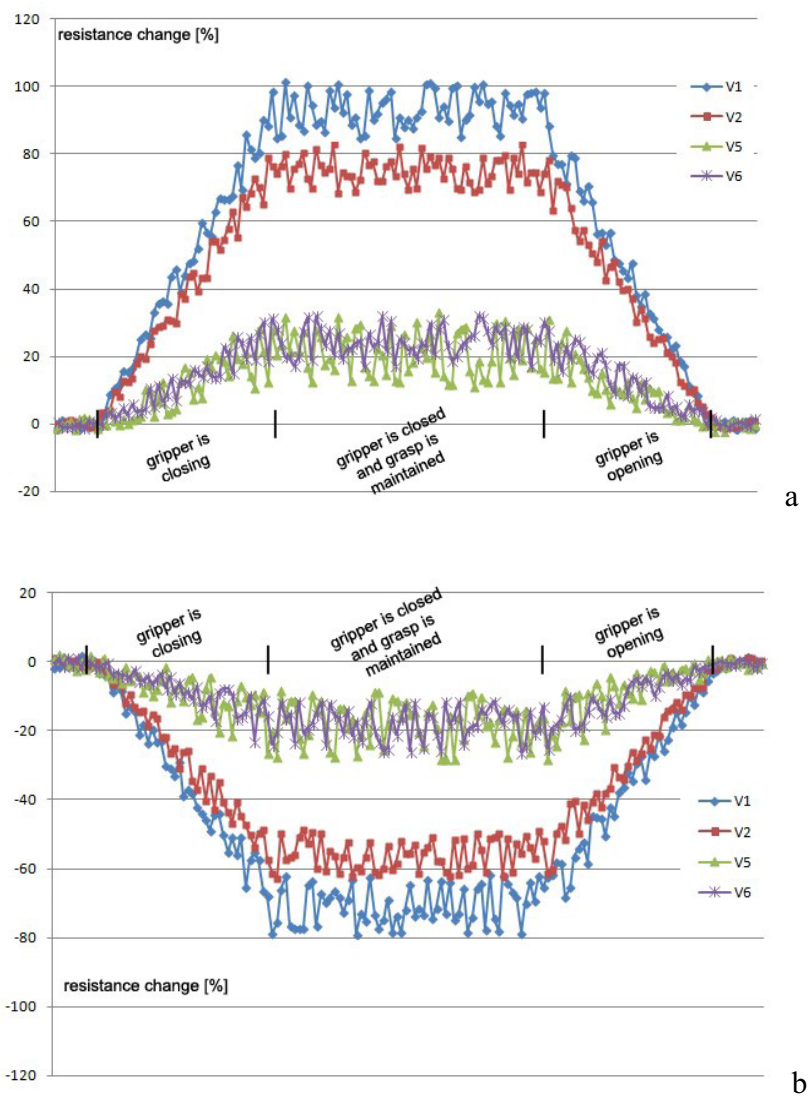

Fig. 9. Relative conductive circuit resistance during work cycle: $a$ - bend sensor embedded in on the finger face coming in contact with the target object; $b$-bend sensor embedded on the opposite face.

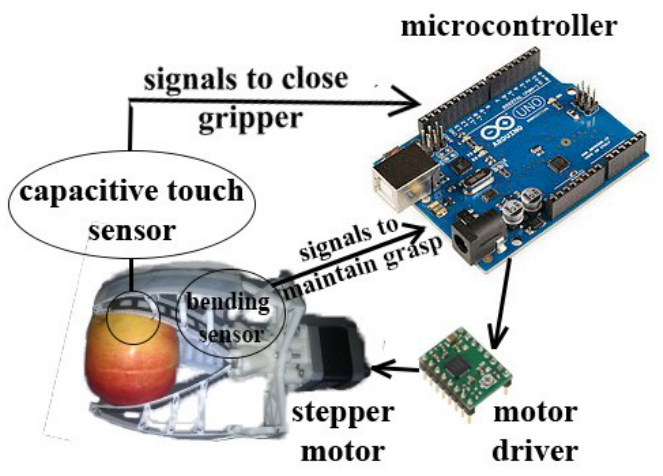

a

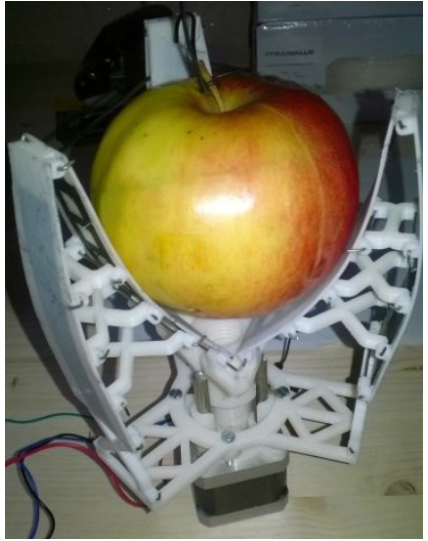

b

Fig. 10. Automated grasping test: $a$-working principle; $b$ - automated grasping an apple. 


\section{Conclusions}

The current paper was focused on developing and testing an adaptive end-effector with three fingers with Fin-ray geometry (Festo) manufactured using new conductive polymers printed inside the fingers' plates - multi-material 3D Printing.

The development process consisted in designing and analyzing different types of conductive circuit patterns, designing each finger and then the whole end-effector, as well as manufacturing these parts from different materials using filament deposition based 3Dprinting process. For the conductive plates, four types of deposition patterns were tested and comparatively analyzed in order to determine the optimal build orientation, i.e. the one ensuring the best electrical properties for the application.

Thus, two experimental stands were designed, manufactured and used. They were needed for: (1) finding suitable build patterns and orientation for 3D printing end-effector fingers with embedded bend sensing capabilities, (2) determining which conductive circuit design performs better and (3) checking the gripper prototype's performance in a 12-hour endurance test (successive closing and releasing fingers for grasping a tennis ball, 8 seconds/cycle).

The results of the bending test showed that the conductive circuit printed horizontally in an orthogonal pattern rotated by $45^{\circ}$ ensures the best bend sensing capabilities, being preffered to the vertical builds.

The results of the endurance test showed good repeatability for 4 of the 6 tested conductive circuit designs. The design that uses a line pattern (v1) had the most stable signal throughout the 24 hours of continous testing. This sensor design was then succesfully used in an automated grasping procedure that uses input signals from the embeded capacitive touch sensor and bending sensor.

\section{References}

1. A.T. Gaynor, N.A. Meisel, C.B. Williams, J.K. Guest, J. Manuf. Sci. Eng., 136, 061015 (2014)

2. J. Scott et al., Additive Manufacturing: Status and Opportunities, (Science and Technology Policy Institute 2012)

3. A. Ion et al., Proceedings of UIST '16, Tokyo, Japan (available at: http://alexandraion.com/wpcontent/uploads/2016UIST-Metamaterial-Mechanisms-authors-copy.pdf, 2016)

4. S. Tammas-Williams, I. Todd, SMM-11342 (available at: http://dx.doi.org/10.1016/j.scriptamat.2016.10.030, 2016)

5. T.M. Llewellyn-Jones et al., Smart Mater. Struct., 25( 02LT01), (available at: http://iopscience.iop.org/article/10.1088/0964-1726/25/2/02LT01, 2016)

6. E. Brown et al., PNAS, 107 (44), 18809 (2010)

7. P.G. Madden, G.V. Lauder, Silas Alben, J. R. Soc. Interface, 4 (13), 243 (2007)

8. $* * *$ https://www.festo.com/net/en-gb_gb/SupportPortal/press.aspx?DocumentId=367236, Accessed 20.9.2016

9. W. Crooks, G. Vukasin, M. O'Sullivan, W. Messner, and C. Rogers, Front. Robot. AI, 3(70) (available at: doi: 10.3389/frobt.2016.00070, 2016)

10. K.C. Galloway, K.P. Becker, B. Phillips, J. Kirby, S. Licht, D. Tchernov, et al., Soft Robot, 3(1), $23(2016)$

11. P. Maeder-York, T. Clites, E. Boggs, R. Neff, P. Polygerinos, D. Holland, et al., J. Med Dev, 8(2), 020933 (2014)

12. A. Zapciu, G. Constantin, Manufacturing Systems, 11(4), 201 (2016) 